\title{
THE GEOENGINEERING DILEMMA: TO SPEAK OR NOT TO SPEAK
}

\author{
An Editorial Comment
}

An intriguing dichotomy has developed within the field of atmospheric and climate research. On the one side, it has become common practice to examine pessimistic future scenarios of anthropogenic pollutant emissions and their environmental impacts. Not surprisingly, compared to the alternative "best guess" or "maximum feasible reduction" emissions scenarios, the pessimistic scenario simulations tend to predict large changes in the climate system and air quality. These scenarios can certainly attract attention to the possibly disastrous consequences of a careless environmental stewardship. However, they can also backfire politically, being seen as "unrealistic scare tactics" or "Hollywood horrors" put forth by scientists with environmentalist agendas. Furthermore, each simulation can only show one potential outcome among many possible states for a strongly perturbed climate. The predicted extreme outcomes of pessimistic scenarios will tend to vary widely from model to model. Unless this is communicated effectively, it can lead to confusion among policy makers, and can reduce confidence in such predictive studies. Nevertheless, such pessimistic scenario calculations are not only allowed, but are strongly condoned, for instance by the IPCC (2001), which employs these types of pessimistic scenarios as a central part of its regular assessments. This is well justified, given the most important outcome of these scenario calculations: scientists learn, and they learn a lot about the behavior of the earth system. The key is ensuring that the results are reported to the public and policy-making sectors as clearly and responsibly as possible, which is part of the purpose of the intense IPCC review procedure.

On the other side of the dichotomy, serious scientific research into geoengineering possibilities, such as discussed in the publications by Crutzen (2006) and Cicerone (2006), is not at all condoned by the overall climate and atmospheric chemistry research communities. Quite the contrary, according to Cicerone, "refereed publications that deal with such ideas are not numerous nor are they cited widely". In the discussions that surrounded the drafting of Crutzen's article, there was a passionate outcry by several prominent scientists claiming that it is irresponsible to publish such an article focused on a particular geoengineering proposal. Such critical responses to discussions on geoengineering have a long history, as discussed by Schneider (2001).

Why would I compare pessimistic emissions scenarios with studies of geoengineering, when it is clear that there are important differences between these? Growing emissions are an unintentional by-product of the striving for comfort and prosperity; in contrast, geoengineering is a contemplated reaction to prevent a perceived impending catastrophe. But cannot these also be seen as two different forms 
of pessimistic future scenarios? Both have implications for major changes in the climate and atmospheric composition: one is a "secondary" perturbation, arising from increasing rates of fossil fuel, biofuel and biomass burning, while the other is a "primary" perturbation, in terms of geoengineering which may eventually be undertaken as a reaction to our failure to otherwise control emissions.

I deeply hope that we will never see an era where widespread geoengineering like that discussed by Crutzen is being practiced, but there is no way to guarantee that this will be the case. Crutzen expresses the sincere concern that we may eventually reach the state of extreme climate change where the overall international sentiment is in favor of applying geoengineering. If we do not conduct careful research now, we will not be prepared to advise politicians on how to best approach large-scale geoengineering applications - including providing sound information on the various risks involved, and on which ideas should not be pursued further. I would add to this a further concern: it is easy to imagine a future scenario in which certain nations begin to undertake large-scale geoengineering efforts on their own. It is not uncommon for nations to act unilaterally in what they perceive as their own best interests, regardless of any international outcry about the consequences for the rest of the world. It does not take much effort to scan the literature and internet and quickly come up with a variety of climate-change-mitigating geoengineering proposals. However, it does take extensive knowledge and diligent research to assess the effectiveness, economic and technological requirements, and possible unintended consequences of each of these. Geoengineering efforts will probably always be accompanied by a questionable effectiveness and sizeable risks. This will be compounded if current research is not supported or is widely condemned. In that case, any future geoengineering undertakings, whether multi-national or unilateral, will be based on the poorly-informed best guesses of national policymakers and their advisors. There would be little international knowledge of what likely consequences to anticipate and what potential side effects to monitor for. Furthermore, without a good overview of potential geoengineering efforts which might eventually be undertaken, it would be difficult to monitor for the possibility of "covert" geoengineering. I am in support of Cicerone's recommendation of a moratorium on large-scale geoengineering applications. It will be very important to consider who can enforce such a moratorium and how they will do it. It may also be sensible to add to Cicerone's recommendation a further general moratorium against open-market economic gain from geoengineering applications, as discussed by Chisholm et al. (2001) and Lawrence (2002) with respect to proposals for $\mathrm{CO}_{2}$ reduction through oceanic iron fertilization. Furthermore, a clear line will need to be drawn between allowed scientific experiments which are small-scale yet large enough to have statistically significant signals, and what goes beyond this, so that "science" cannot be used as camouflage for unilateral attempts to undertake largescale geoengineering efforts.

Thus the pertinent question is not "To speak or not to speak?", but rather "How to speak?" about geoengineering. One part of this is focusing on scientific issues, and, 
to the extent possible, avoiding blanket judgment terminology such as "good" and "bad". Scientifically, what is quantifiable is change, variability, and stability (where the distinction between change and variability often depends on the time scale). Whether change and variability are good or bad depends on your particular situation, as already pointed out by Schneider (2001): "anything other than a preservation of current structure and function demands a definition of 'improvement', and this judgement will be very different across diverse cultures." This applies not only to the relatively short-term interests of present human cultures, but also to much longer time scales: it is likely that climate change and variability are important factors influencing genetic diversity and evolution, including human evolution, though these relationships appear to be quite complex (e.g., Behrensmeyer, 2006; Hadly et al., 2004).

Another part of how to speak about geoengineering is captured by Cicerone's plea for support of careful, conscientious, and scientifically sound research into geoengineering proposals, following the standard scientific path of publication in peer-reviewed journals. Vigilence is required in the manner in which we discuss geoengineering in the scientific literature. This is especially a responsibility of editors, who can filter incoming literature and solicit useful discussions, as well as of referees, who need to be aware of their importance as "watchdogs" in this very sensitive issue. Further, scientists will need to be particularly cautious about making conclusions and predictions based on current models, which are known to be in need of significant further development. Perhaps, as I have heard suggested in recent discussions, the additional filtering of such results could be considered as a future task for the IPCC assessments, providing an appropriate forum for further community assessment of the peer-reviewed literature.

Many concerns have been expressed about the possible future application of widespread geoengineering. Especially worrisome is that it could end up being used as an excuse for not needing to reduce greenhouse gas emissions, in which case it follows that the intensity of any geoengineering efforts (e.g., the amount of sulfur injected into the stratosphere per year) would also need to increase to keep pace with accumulating, long-lived greenhouse gases. Nevertheless, geoengineering is being discussed intensely, at least outside of the formal scientific literature, and it is not going to go away by ignoring it or refusing to discuss it scientifically. Thus, I would argue that it is our responsibility to do what scientists do best: explore it, understand it deeply, and eventually describe the key points of this understanding in terms accessible to the educated public and policy-making communities, in order to support well-informed decisions on geoengineering in the future.

\section{References}

Behrensmeyer, A. K.: 2006, 'Climate change and human evolution', Science 311, 476-478. Chisholm, S. W., Falkowski, P. G., and Cullen, J. J.: 2001, 'Dis-crediting ocean fertilization', Science 294, 309-310. 
Cicerone, R. J.: 2006, 'Geoengineering: Encouraging research and overseeing implementation', Climatic Change, this issue, DOI: 10.1007/s10584-006-9102-x.

Crutzen, P. J.: 2006, 'Albedo enhancement by stratospheric sulfur injections: A contribution to resolve a policy dilemma?', Climatic Change, this issue, DOI: 10.1007/s10584-006-9101-y.

Hadly, E. A., Ramakrishnan, U., Chan, Y. L., van Tuinen, M., O’Keefe, K., Spaeth, P. A., and Conroy, C. J.: 2004, 'Genetic response to climatic change: Insights from ancient DNA and phylochronology', PLoS Biol 2:e290.

IPCC: 2001, 'Climate Change 2001, in Houghton, J. T., et al. (eds.), The Scientific Basis, Third Assessment Report of the Intergovernmental Panel on Climate Change', Cambridge University Press, Cambridge, UK and New York, N.Y., U.S.A.

Lawrence, M. G.: 2002, 'Side effects of oceanic iron fertilization', Science 297, 1993.

Schneider, S. H.: 2001, 'Earth systems engineering and management', Nature 409, 417-421.

Max Planck Institute for Chemistry

MARK G. LAWRENCE

Atmospheric Chemistry Department

Postfach 3060

55020 Mainz, Germany

E-mail:lawrence@mpch-mainz.mpg.de 